\title{
INTERAÇÃO DE GLYPHOSATE COM CARFENTRAZONE-ETHYL ${ }^{1}$
}

\author{
Glyphosate - Carfentrazone-ethyl Interaction
}

WERLANG, R.C. ${ }^{2}$ e SILVA, A.A. ${ }^{3}$

\begin{abstract}
RESUMO - Foi conduzido um experimento em condições controladas para determinar a interação do carfentrazone-ethyl em mistura no tanque com o herbicida glyphosate, no controle de seis espécies de plantas daninhas. Glyphosate aplicado isoladamente na dose de $720 \mathrm{~g} \mathrm{ha}^{-1}$ foi eficaz no controle de Amaranthus hybridus (100\%), Desmodium tortuosum (100\%), Bidens pilosa (99\%), Eleusine indica (96\%), Digitaria horizontalis (100\%) e Commelina benghalensis (93\%) aos 21 DAA. Carfentrazone-ethyl aplicado isoladamente controlou eficazmente $C$. benghalensis. As misturas de glyphosate nas doses de 252 e $720 \mathrm{~g} \mathrm{ha}^{-1}$ com carfentrazone-ethyl nas doses de 15 e $30 \mathrm{~g} \mathrm{ha}^{-1}$ demonstraram efeito aditivo no controle de A. hybridus, D. tortuosum e Bidens pilosa, à exceção das misturas de glyphosate na dose de $252 \mathrm{~g} \mathrm{ha}^{-1}$ com as doses de $15 \mathrm{e}$ $30 \mathrm{~g} \mathrm{ha}^{-1}$ de carfentrazone-ethyl, que proporcionam efeito sinergístico no controle de $D$. tortuosum. A adição das duas doses de carfentrazone-ethyl antagonizou o efeito de glyphosate na menor dose (252 $\left.\mathrm{g} \mathrm{ha}^{-1}\right)$ no controle de $E$. indica, apresentando, no entanto, efeito aditivo com o glyphosate na maior dose $\left(720 \mathrm{~g} \mathrm{ha}^{-1}\right)$. Já para $D$. horizontalis, as misturas de carfentrazone-ethyl com glyphosate na menor dose (252 $\left.\mathrm{g} \mathrm{ha}^{-1}\right)$ apresentaram efeito sinergístico no controle dessa espécie, demonstrando, ainda, efeito aditivo na mistura com glyphosate na dose de $720 \mathrm{~g} \mathrm{ha}^{-1}$. A mistura de carfentrazone-ethyl com glyphosate proporcionou efeito aditivo no controle de $C$. benghalensis, independentemente das combinações de doses avaliadas. Os resultados deste experimento indicam que carfentrazone-ethyl apresenta comportamento diferenciado quanto à interação com glyphosate, dependendo da espécie de planta daninha e da dose dos herbicidas utilizados na mistura em tanque, sendo complementar na mistura em tanque com glyphosate, pois demonstrou efeito antagônico em poucas das combinações estudadas, prevalecendo seu efeito aditivo na mistura com glyphosate, no controle das espécies avaliadas.
\end{abstract}

Palavras-chave: herbicida, antagonismo, sinergismo, mistura em tanque.

ABSTRACT - Under evironmental controlled conditions experiments were conducted to determine the efficacy of carfentrazone-ethyl in tank mixture with glyphosate to control six weed species. Glyphosate alone at $720 \mathrm{~g} \mathrm{ha}^{-1}$ was effective in controlling Amaranthus hybridus (100\%), Desmodium tortuosum (100\%), Bidens pilosa (99\%), Eleusine indica (96\%), Digitaria horizontalis (100\%) and Commelina benghalensis (93\%) at 21 DAA. Carfentrazone-ethyl alone controlled only C. benghalensis. efficiently. Glyphosate mixtures (252 and $720 \mathrm{~g} \mathrm{ha}^{-1}$ ) with carfentrazone-ethyl at 15 and $30 \mathrm{~g} \mathrm{ha}^{-1}$ showed an additive effect in controlling $\boldsymbol{A}$. hybridus, D. tortuosum and Bidens pilosa, except for glyphosate mixture at $252 \mathrm{~g} \mathrm{ha}^{-1}$ with carfentrazone-ethyl at 15 and $30 \mathrm{~g} \mathrm{ha}^{-1}$, which showed synergism in controlling $\boldsymbol{D}$. tortuosum. The addition of the two carfentrazone-ethyl doses antagonized the effect of the smallest dose $\left(252 \mathrm{~g} \mathrm{ha}^{-1}\right)$ of glyphosate on $\boldsymbol{E}$. indica control. However, the largest glyphosate dose $\left(720 \mathrm{~g} \mathrm{ha}^{-1}\right)$ provided additive effect when mixed with both carfentrazone-ethyl doses. For $\boldsymbol{D}$. horizontalis, carfentrazone-ethyl mixtures with glyphosate in the smallest dose $\left(252 \mathrm{~g} \mathrm{ha}^{-1}\right)$ had a synergistic effect in the control of this species, also showing and additive effect in the mixture with glyphosate at $720 \mathrm{~g} \mathrm{ha}^{-1}$. Carfentrazone-ethyl mixture with glyphosate provided an additive effect in $\mathbf{C}$. benghalensis control, regardless of dose combinations. The results of this experiment indicate that carfentrazone-ethyl presents a differentiated behavior when interacting with glyphosate, depending on the weed species and the herbicide dose used in the tank mixtures, being complementary in tank-mix combinations with glyphosate, since it showed an antagonistic effect in few combinations, with its additive effect prevailing in the mixture with glyphosate for the control of the evaluated species.

Key words: herbicide, antagonism, synergism, tank mixture.

1 Recebido para publicação em 6/11/2001 e na forma revisada em 15/3/2002.

2 Mestrando, Dep. de Fitotecnia da Universidade Federal de Viçosa-UFV, 36571-000 Viçosa-MG; ${ }^{3}$ Prof. do Dep. de Fitotecnia da UFV. 


\section{INTRODUÇÃO}

A introdução de culturas com tolerância ao glyphosate (Delannay et al., 1995) possibilitou novas opções de controle de plantas daninhas em pós-emergência. O glyphosate, por exercer controle de grande número de plantas daninhas mono e dicotiledôneas, perenes e anuais, sendo ambientalmente pouco agressivo, é o herbicida mais conhecido no mundo (Malik et al., 1989). Pertence ao grupo dos inibidores da síntese de aminoácidos e contém o N-(phosphonomethyl) glycina como ingrediente ativo (Rodrigues \& Almeida, 1998). Além disso, o glyphosate é sistêmico, não-seletivo, altamente solúvel em água e seu mecanismo de ação baseia-se na interrupção da rota do ácido chiquímico, responsável pela produção dos aminoácidos aromáticos fenilalanina, tirosina e triptofano, que são essenciais para a síntese de proteínas e divisão celular nas regiões meristemáticas da planta (Hess, 1994).

$\mathrm{O}$ uso repetitivo de um mesmo herbicida ou de diferentes herbicidas com o mesmo mecanismo de ação em uma área favorece a seleção de espécies de plantas daninhas resistentes a esses produtos (Christoffoleti et al., 1994). Mesmo sendo classificado como um herbicida de ação total, diversas plantas daninhas são relatadas como tolerantes a glyphosate (Kapusta et al., 1994; Jordan et al., 1997). No Brasil, Durigan et al. (1988), Galli (1991) e Santos et al. (2001) relatam a tolerância de espécies de trapoeraba. Vargas et al. (1999) especulam que a elevada tolerância das espécies do gênero Commelina ao glyphosate esteja relacionada com a insensibilidade da enzima EPSPs dessas espécies ao herbicida. Todavia, Santos et al. (2001) sugerem que diferenças na tolerância ao glyphosate entre espécies de trapoeraba estejam relacionadas a diferenças morfológicas e fisiológicas, que possibilitam maior ou menor translocação do herbicida na planta.

Uma das estratégias de controle das espécies tolerantes ao glyphosate é o seu uso em mistura com outros herbicidas seletivos (para culturas tolerantes ao glyphosate), aumentando o espectro de ação. A mistura de chlorimuronethyl com glyphosate melhorou o controle de Ipomoea hederaceae e Sesbania exaltata, comparado com glyphosate isolado (Vidrine et al., 1997). Segundo Lich et al. (1997), a mistura de bentazon com glyphosate proporcionou melhor controle de Chenopodium album do que glyphosate isolado.

A mistura de glyphosate com outros herbicidas tem demonstrado resultados de antagonismo ou sinergismo. Chlorimuron foi compativel com glyphosate, porém o acifluorfen demonstrou atividade antagônica no controle de Echinichloa crus-galli (Jordan et al., 1997). A mistura de pequenas doses de lactofen com glyphosate age sinergisticamente no controle de Malva parviflora (Wells \& Appleby, 1992). Já misturas de lactofen em doses maiores provocam efeito antagônico, aparentemente pela destruição da membrana plasmática, ocasionando menor absorção de glyphosate. No entanto, a mistura de oxyfluorfen com glyphosate melhorou o controle de Cyperus esculentus, pois promoveu maior absorção de ${ }^{14} \mathrm{C}$-glyphosate por esta espécie (Pereira \& Crabtree, 1986).

A mistura de glyphosate com acifluorfen ou bentazon proporcionou melhor controle de dicotiledôneas; entretanto, o controle de gramíneas foi prejudicado em algumas condições, quando glyphosate foi usado em mistura com outros herbicidas (Hydrick \& Shaw, 1994; Selleck \& Baird, 1981).

Segundo Starke \& Oliver (1998), a mistura em tanque de glyphosate com chlorimuron foi caracterizada como aditiva e não reduziu a absorção e translocação de ${ }^{14} \mathrm{C}$-glyphosate em Echinichloa crus-galli, Ipomoea lacunosa e Abutilon theophrasti. Já as misturas de fomesafen com glyphosate foram antagônicas no controle dessas espécies, reduzindo a absorção e translocação de glyphosate. Sulfentrazone em mistura no tanque com glyphosate proporcionou efeito antagônico no controle de Echinichloa crus-galli, Amaranthus palmeri, Eleusine indica e Ipomoea hederaceae var. integriuscula.

Outros herbicidas seletivos que controlam plantas daninhas tolerantes ao glyphosate precisam ser estudados quanto à compatibilidade de suas misturas com esse ingrediente ativo, devido à necessidade de controle dessas espécies daninhas no manejo em culturas com tolerância ao glyphosate e, ainda, por proporcionar outras opções no controle de espécies "problemas" em dessecação para plantio direto. Alguns produtos estão sendo estudados para substituição do 2,4-D, na mistura com glyphosate ou sulfosate na dessecação, objetivando a 
redução do período de espera antes do plantio, como: sulfentrazone, flumioxazin e carfentrazone-ethyl (Gazziero et al., 2000; Silva \& Cobucci, 2000; Garcia \& Nascimento, 2000).

O carfentrazone-ethyl é um herbicida do grupo químico das aril triazolinonas, cujo mecanismo de ação consiste na inibição da formação da enzima protoporfirinogênio oxidase (PPO), que participa na síntese da clorofila. É registrado no Brasil para as culturas de milho, soja, algodão, café e citros, possuindo excelente controle de plantas daninhas dicotiledôneas e especialmente Commelina benghalensis e Ipomoea grandifolia (Corrêa \& Borges, 2000; Oliveira Jr. et al., 2000; FMC Corp., 2001). Corrêa \& Borges (2000) relatam que carfentrazone-ethyl apresenta efeito sinergístico quando misturado ao glyphosate no controle de Commelina benghalensis.

O objetivo do presente trabalho foi determinar o tipo de interação na mistura em tanque de glyphosate com carfentrazone-ethyl no controle de Commelina benghalensis, Bidens pilosa, Eleusine indica, Desmodium tortuosum, Amaranthus hybridus e Digitaria horizontalis.

\section{MATERIAL E MÉTODOS}

O experimento foi realizado em casa de vegetação, no período de março a maio de 2001.
O substrato utilizado foi um Argissolo Vermelho-Amarelo Câmbico, fase terraço. Commelina benghalensis, Bidens pilosa, Eleusine indica, Desmodium tortuosum, Amaranthus hybridus e Digitaria horizontalis foram semeadas em bandejas plásticas, contendo solo previamente adubado, sendo posteriormente transplantadas para vasos com $6,0 \mathrm{~kg}$ de substrato. A unidade experimental foi constituída por um vaso contendo duas plantas de cada espécie. A irrigação dos vasos ocorreu diariamente, procurando manter o solo próximo à capacidade de campo, sendo ainda realizadas adubações de cobertura periodicamente. Durante a condução do experimento anotaram-se diariamente as temperaturas máxima e mínima e a umidade relativa do ar, no interior da casa de vegetação (Figura 1).

Os tratamentos descritos na Tabela 1 foram aplicados 40 dias após a emergência, quando as espécies apresentavam as seguintes condições de desenvolvimento: Commelina benghalensis ( 8 pares de folhas e $20 \mathrm{~cm}$ ), Bidens pilosa (10 pares de folhas, $35 \mathrm{~cm}$ e início do florescimento), Eleusine indica (perfilhada com 9 folhas e $40 \mathrm{~cm}$ ), Desmodium tortuosum ( 8 pares de folhas e $25 \mathrm{~cm}$ ), Amaranthus hybridus (13 pares de folhas, $49 \mathrm{~cm}$ e em floração) e Digitaria horizontalis (perfilhada, com 9 folhas e $40 \mathrm{~cm}$ ).

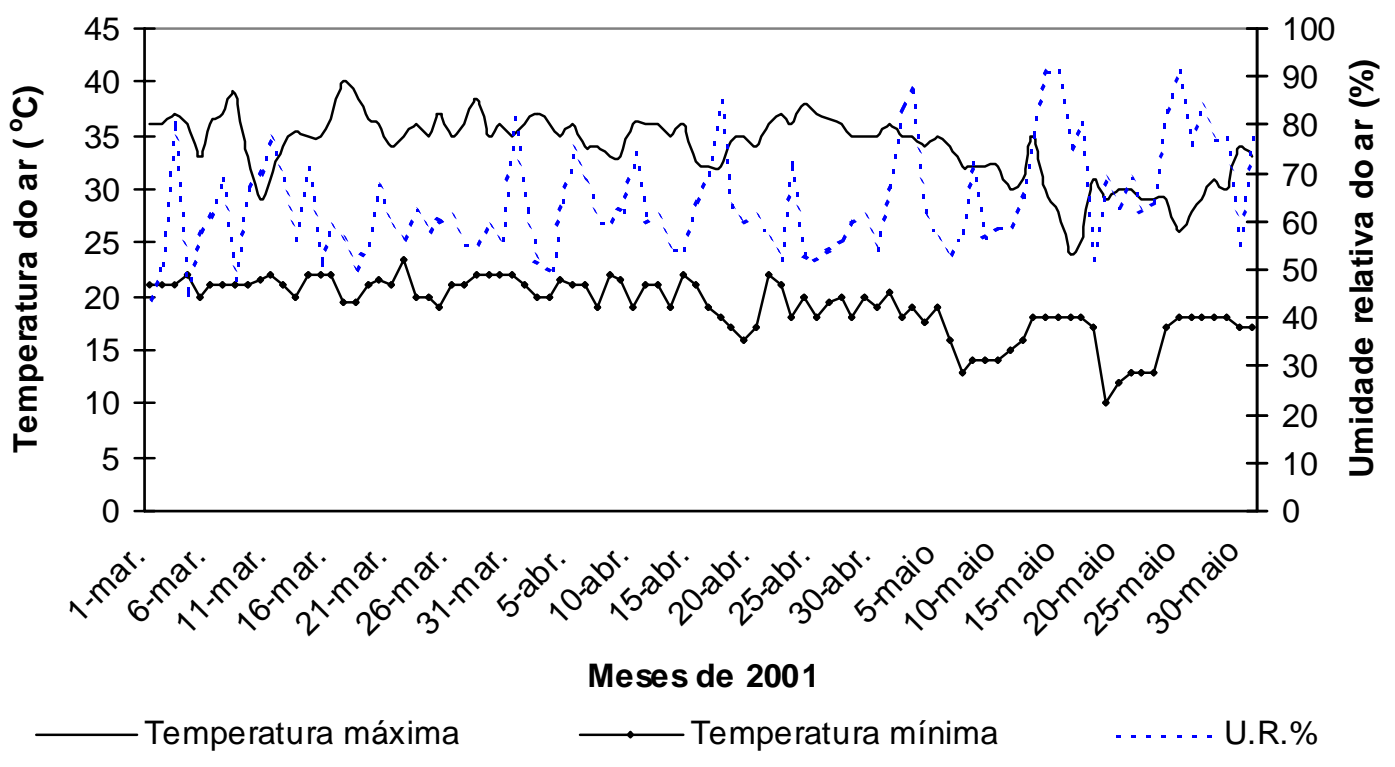

Figura 1 - Dados meteorológicos observados no interior da casa de vegetação, durante o período de realização do experimento. Viçosa-MG, 2001. 
Tabela 1 - Tratamentos avaliados no estudo da interação de glyphosate com carfentrazone-ethyl no controle de plantas daninhas. Viçosa-MG, 2001

\begin{tabular}{|c|c|c|c|}
\hline \multicolumn{2}{|c|}{ Produtos } & \multicolumn{2}{|c|}{ Doses } \\
\hline Nome comum & Nome comercial & i.a. e/ou eq. ac. em g ha ${ }^{-1}$ & P.C. $\mathrm{g} \mathrm{ha}^{-1}$ ou $\mathrm{mL} \mathrm{ha}{ }^{-1}$ \\
\hline $\begin{array}{l}\text { Glyphosate } \\
\text { Glyphosate } \\
\text { Carfentrazone-ethyl } \\
\text { Carfentrazone-ethyl } \\
\text { Glyphosate + carfentrazone-ethyl } \\
\text { Glyphosate + carfentrazone-ethyl } \\
\text { Glyphosate + carfentrazone-ethyl } \\
\text { Glyphosate + carfentrazone-ethyl }\end{array}$ & $\begin{array}{l}\text { Roundup }^{\underline{1}} \\
\text { Roundup } \\
\text { Aurora }^{2} l \\
\text { Aurora } \\
\text { Roundup + Aurora } \\
\text { Roundup + Aurora } \\
\text { Roundup + Aurora } \\
\text { Roundup + Aurora }\end{array}$ & $\begin{array}{c}252 \\
720 \\
15 \\
30 \\
252+15 \\
252+30 \\
720+15 \\
720+30\end{array}$ & $\begin{array}{c}700 \\
2.000 \\
37,5 \\
75,0 \\
700+37,5 \\
700+75,0 \\
2.000+37,5 \\
2.000+75,0\end{array}$ \\
\hline Testemunha & -- & -- & -- \\
\hline
\end{tabular}

A aplicação dos tratamentos foi feita com um pulverizador costal pressurizado com $\mathrm{CO}_{2}$, equipado com uma barra possuindo dois bicos XR-110.03, mantendo-se a pressão constante em 2,5 kgf cm${ }^{-2}$, aplicando-se $200 \mathrm{~L} \mathrm{ha}^{-1} \mathrm{de}$ calda. As aplicações foram realizadas pela manhã (das 8 às 9h), estando as plantas em condições de solo úmido, com umidade relativa de $76 \%$, temperatura ambiente de $26{ }^{\circ} \mathrm{C}$ e velocidade do ar menor que $4 \mathrm{~km} \mathrm{~h}^{-1}$.

Foi utilizado o delineamento experimental inteiramente casualizado, com quatro repetições, com os tratamentos dispostos em esquema fatorial $(3 \times 3)$, sendo constituído por três níveis de glyphosate (zero, 252,0 e 720,0 $\mathrm{g} \mathrm{ha}^{-1}$ ) e três níveis de carfentrazone-ethyl (zero, 15,0 e 30,0 $\mathrm{g} \mathrm{ha}^{-1}$ ).

A eficiência de controle das espécies estudadas foi avaliada aos 3, 7, 14 e 21 dias após a aplicação (DAA), com base nos sintomas de fitotoxicidade, utilizando a escala em que $0 \%$ correspondeu à ausência de sintomas visíveis e 100\% à morte das plantas. Aos 21 DAA, as plantas foram cortadas rente ao solo, para determinação da biomassa seca por vaso. Para isso, o material vegetal foi secado em estufa de circulação forçada a $70{ }^{\circ} \mathrm{C}$, até adquirir peso constante. A redução da matéria seca foi calculada em relação à testemunha, que foi considerada como $0 \%$ de redução.

Todos os dados avaliados foram submetidos à análise de variância, e para comparação das médias utilizou-se o teste de Tukey a 5\% de probabilidade. Os dados foram também submetidos ao modelo de distribuição multiplicativo
(Colby, 1967), conforme Starke \& Oliver (1998), para estudo das interações entre os herbicidas, sendo utilizada a equação 1 para calcular a resposta esperada:

Equação 1: $\mathrm{E}=100-[((100-\mathrm{x}) *(100-\mathrm{y})) / 100]$

em que $E$ é a redução esperada no crescimento (como a redução na porcentagem de controle esperada ou a redução na matéria seca) e $x$ e $y$ representam a redução no crescimento obtida pelo tratamento com o herbicida $\mathrm{x}$ e $\mathrm{y}$, respectivamente.

As possiveis respostas de interação constituem-se em: a) antagonismo - que é caracterizado pela ação conjunta de dois herbicidas apresentando resposta de um organismo-teste na sua combinação, menor do que a resposta esperada, obtida por modelos apropriados de referência, como o proposto por Colby (1967); b) sinergismo - é a ação cooperada dos dois herbicidas apresentando resposta no organismo-teste, na aplicação conjunta, superior à resposta esperada obtida por modelos apropriados de referência; e c) aditivo - ocorre quando dois herbicidas reagem apresentando resposta no organismo-teste, na aplicação conjunta, igual à resposta esperada obtida por modelos apropriados de referência (Hatzios \& Penner, 1985).

\section{RESULTADOS E DISCUSSÃO}

Glyphosate aplicado isoladamente na maior dose (720 $\left.\mathrm{g} \mathrm{ha}^{-1}\right)$ foi eficaz no controle de Amaranthus hybridus (100\%), Desmodium 
tortuosum (100\%), Bidens pilosa (99\%), Eleusine indica (96\%), Digitaria horizontalis (100\%) e Commelina benghalensis (93\%) aos 21 DAA (Tabelas 2, 3, 4, 5, 6 e 7). Carfentrazone-ethyl aplicado isoladamente controlou de modo eficaz somente $C$. benghalensis, demonstrando controle total da espécie já aos 7 DAA na dose de $15 \mathrm{~g} \mathrm{ha}^{-1}$. Esses resultados foram semelhantes aos observados por Corrêa \& Borges (2000), Oliveira Jr. et al., (2000) e Ronchi et al. (2001), em que carfentrazone-ethyl demonstrou excelente controle dessa espécie.

Glyphosate aplicado isoladamente na menor dose (252 $\mathrm{g} \mathrm{ha}^{-1}$ ) apresentou, aos 21 DAA, controle moderado de A. hybridus e D. horizontalis (91\%) e não-satisfatório para Desmodium tortuosum (71\%), Bidens pilosa (88\%), Eleusine indica (81\%) e Commelina benghalensis (35\%). Já em mistura em tanque com carfentrazone-ethyl na menor dose (15 $\mathrm{g} \mathrm{ha}^{-1}$ ), demonstrou controle eficiente para Amaranthus hybridus (98\%), Desmodium tortuosum (93\%), Bidens pilosa (94\%), Digitaria horizontalis (94\%) e Commelina benghalensis (100\%) aos 21 DAA. Entretanto, essa mistura não foi eficaz no controle de Eleusine indica (69\%), no mesmo período.
Todas as misturas em tanque de glyphosate com carfentrazone-ethyl demonstraram aumento no controle visual aos 3 DAA para as espécies avaliadas, diferindo dos controles obtidos pelo glyphosate ou carfentrazone-ethyl aplicados isoladamente.

As misturas de glyphosate nas doses de 252 e $720 \mathrm{~g} \mathrm{ha}^{-1}$ com carfentrazone-ethyl nas doses de 15 e $30 \mathrm{~g} \mathrm{ha}^{-1}$ demonstraram efeito aditivo, no controle visual aos 21 DAA, de A. hybridus, D. tortuosum e Bidens pilosa, à exceção da mistura de glyphosate na menor dose com as duas doses de carfentrazone-ethyl, que demonstrou efeito sinergístico no controle de D. tortuosum. No entanto, quando a comparação é realizada com as reduções de matéria seca, o controle de $D$. tortuosumé caracterizado como antagônico, em todas as combinações de doses; já para B. pilosa é antagônico somente nas combinações da menor dose de glyphosate com a maior de carfentrazone-ethyl e da maior dose de glyphosate com a menor de carfentrazone-ethyl. As demais combinações de doses demonstraram efeito aditivo no controle desta espécie. A. hybridus apresentou efeito aditivo no seu controle, avaliado pela redução da matéria seca, ocasionado por todas as misturas de glyphosate com carfentrazone-ethyl.

Tabela 2 - Controle visual, redução da matéria seca avaliada aos 21 DAA e esperada (Colby) de Amaranthus hybridus, após aplicação de herbicidas em pós-emergência. Viçosa-MG, 2001

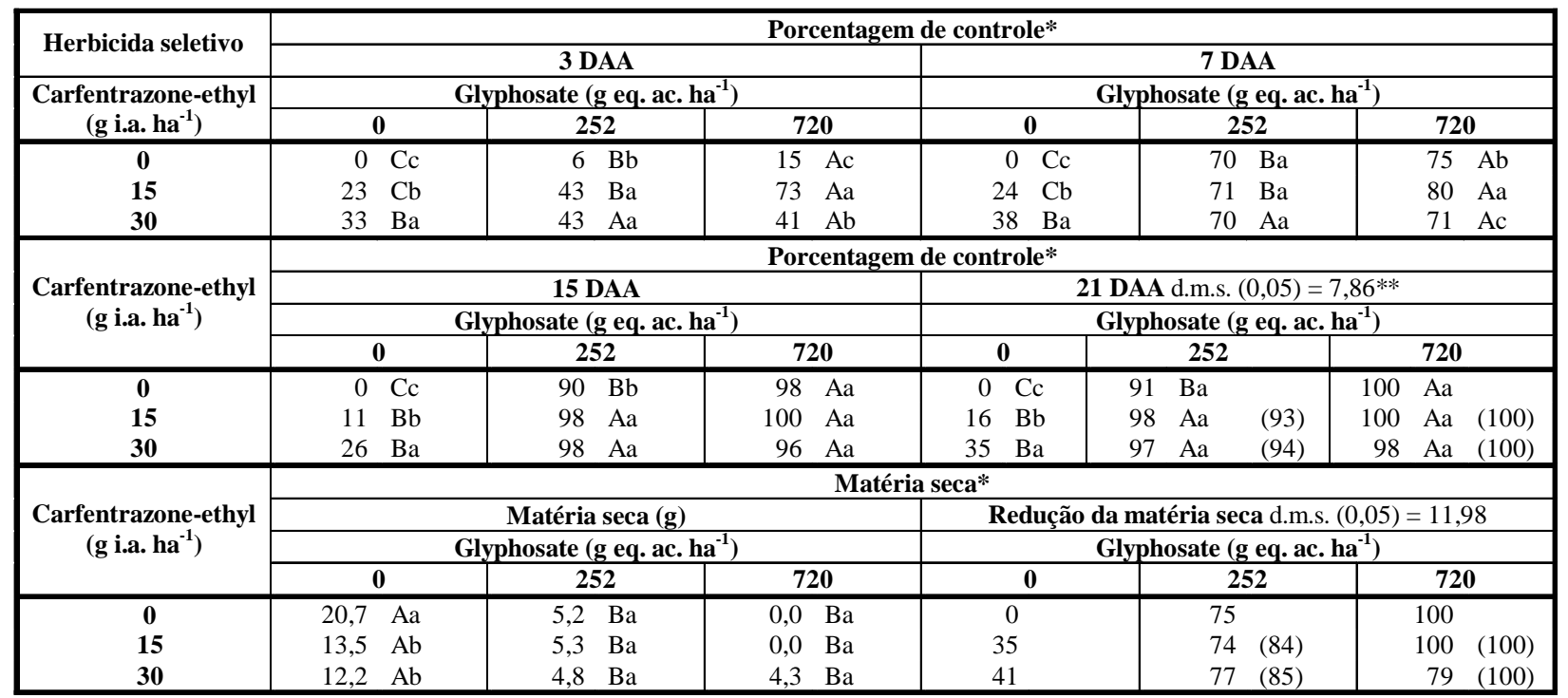

* Médias seguidas pelas mesmas letras, maiúsculas na linha e minúsculas na coluna, não diferem entre si pelo teste de Tukey a $5 \%$ de significância.

** Valores entre parênteses são os valores esperados calculados pela equação de Colby (1967). O sinal negativo após o valor esperado indica efeito antagônico na interação, sinal positivo significa efeito de sinergismo e ausência de sinal indica interação aditiva. 
Tabela 3 - Controle visual, redução da matéria seca avaliada aos 21 DAA e esperado (Colby) de Desmodium tortuosum, após aplicação de herbicidas em pós-emergência. Viçosa-MG, 2001

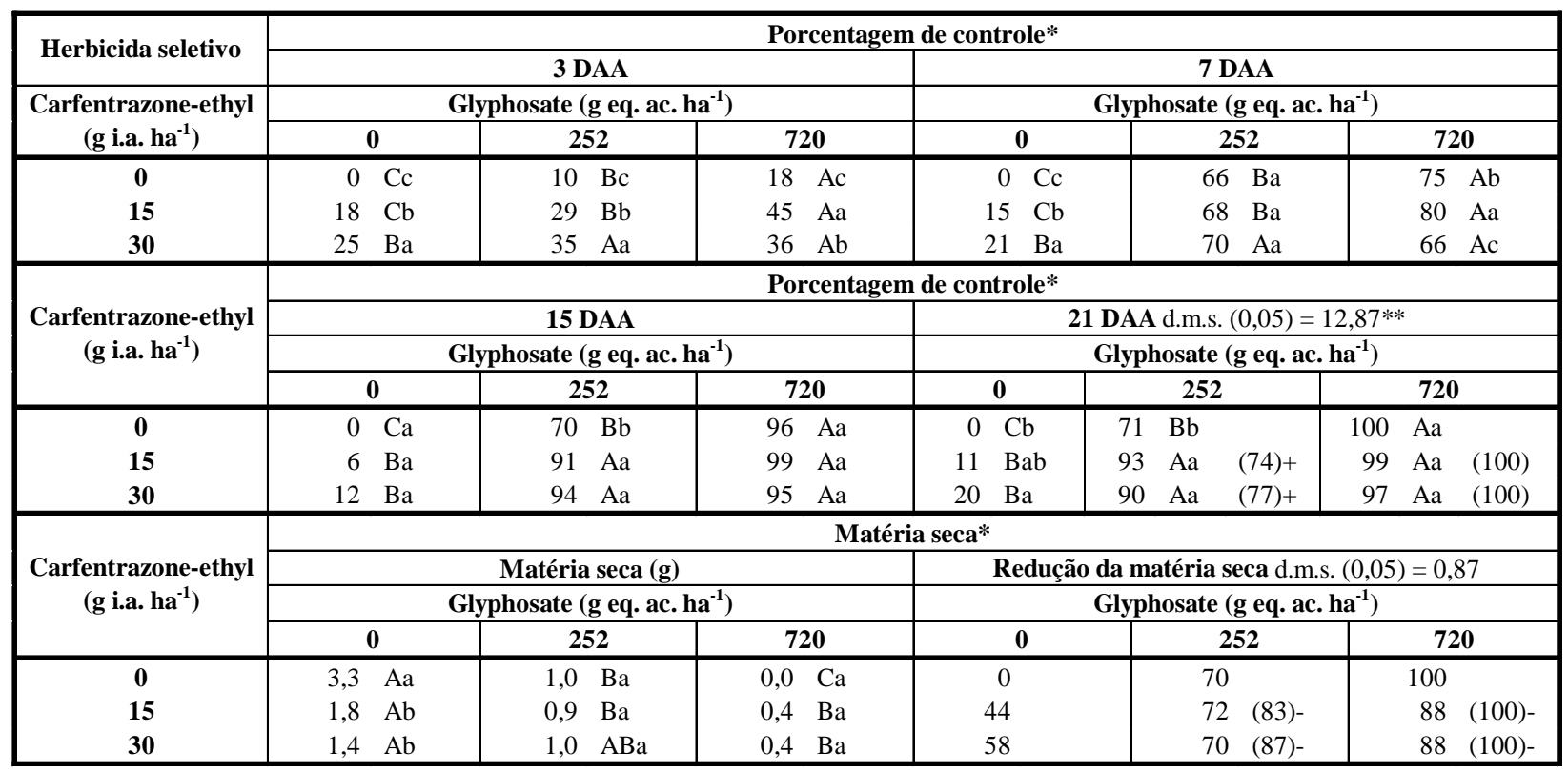

* Médias seguidas pelas mesmas letras, maiúsculas na linha e minúsculas na coluna, não diferem entre si pelo teste de Tukey a $5 \%$ de significância.

** Valores entre parênteses são os valores esperados calculados pela equação de Colby (1967). O sinal negativo após o valor esperado indica efeito antagônico na interação, sinal positivo significa efeito de sinergismo e ausência de sinal indica interação aditiva.

Tabela 4 - Controle visual, redução da matéria seca avaliada aos 21 DAA e esperada (Colby) de Bidens pilosa, após aplicação de herbicidas em pós-emergência. Viçosa-MG, 2001

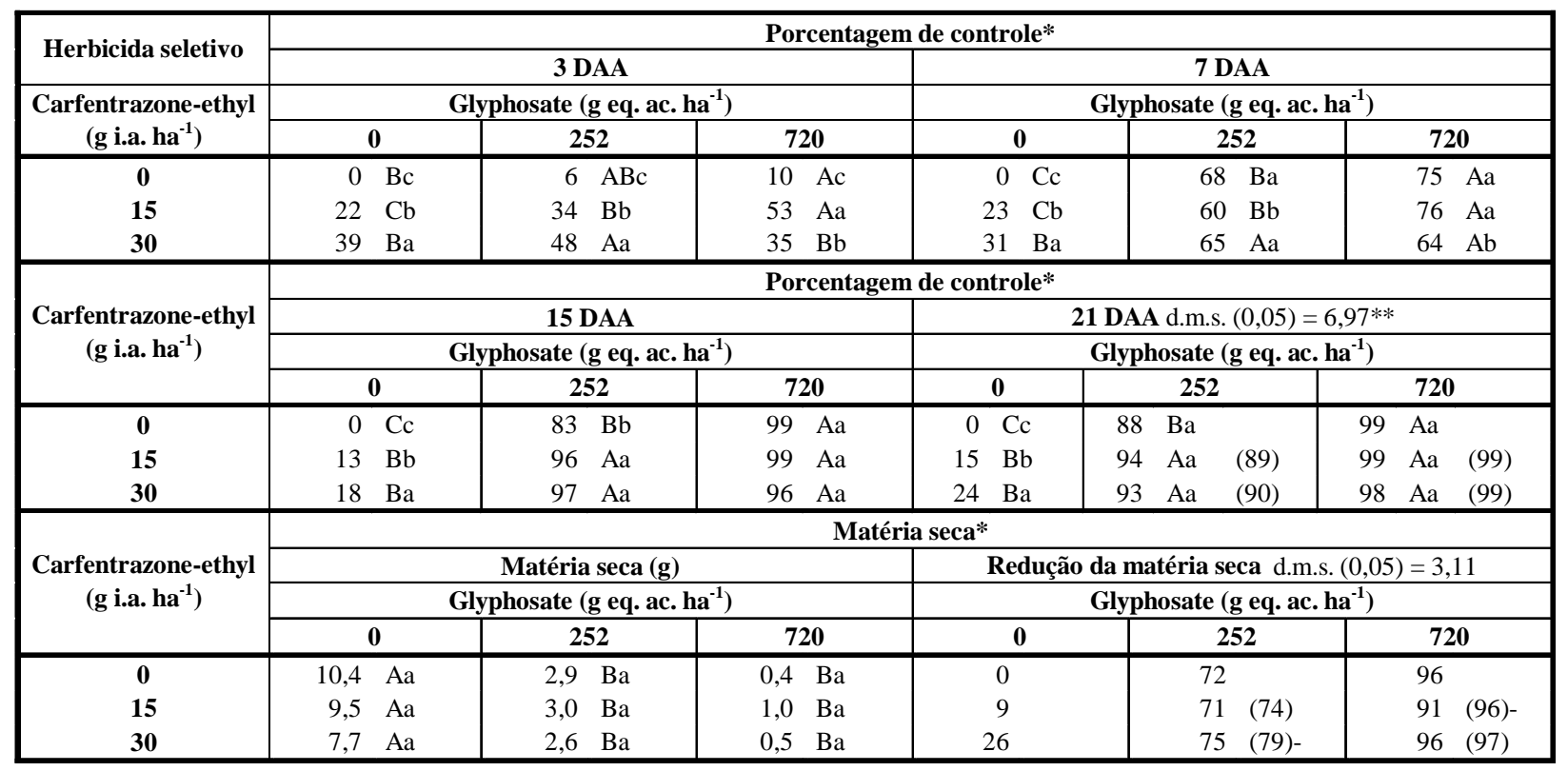

* Médias seguidas pelas mesmas letras, maiúsculas na linha e minúsculas na coluna, não diferem entre si pelo teste de Tukey a $5 \%$ de significância.

** Valores entre parênteses são os valores esperados calculados pela equação de Colby (1967). O sinal negativo após o valor esperado indica efeito antagônico na interação, sinal positivo significa efeito de sinergismo e ausência de sinal indica interação aditiva. 
Tabela 5 - Controle visual, redução da matéria seca avaliada aos 21 DAA e esperada (Colby) de Eleusine indica, após aplicação de herbicidas em pós-emergência. Viçosa, MG, 2001

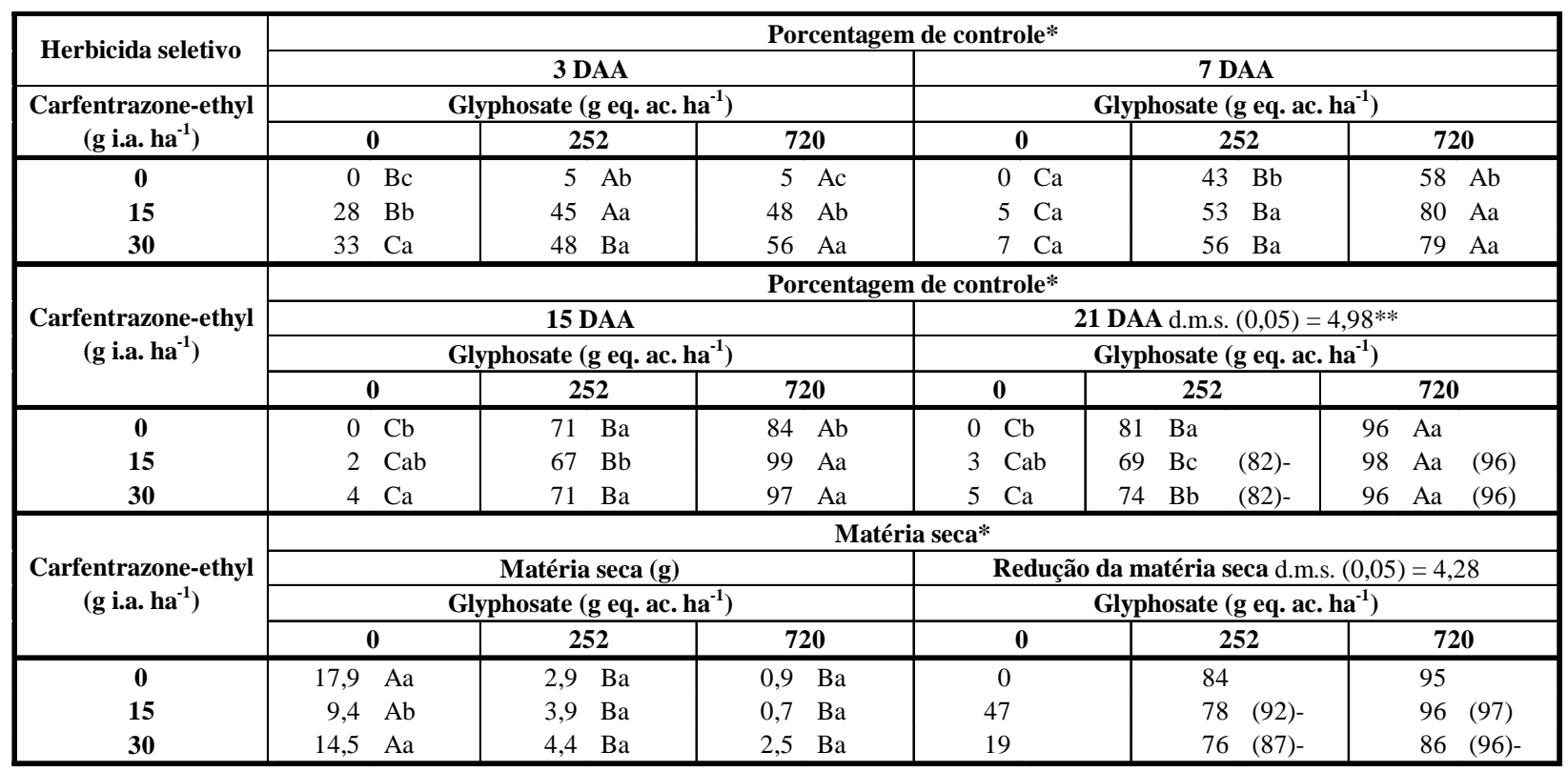

* Médias seguidas pelas mesmas letras, maiúsculas na linha e minúsculas na coluna, não diferem entre si pelo teste de Tukey a $5 \%$ de significância.

** Valores entre parênteses são os valores esperados calculados pela equação de Colby (1967). O sinal negativo após o valor esperado indica efeito antagônico na interação, sinal positivo significa efeito de sinergismo e ausência de sinal indica interação aditiva.

Tabela 6 - Controle visual, redução da matéria seca avaliada aos 21 DAA e esperada (Colby) de Digitaria horizontalis, após aplicação de herbicidas em pós-emergência. Viçosa-MG, 2001

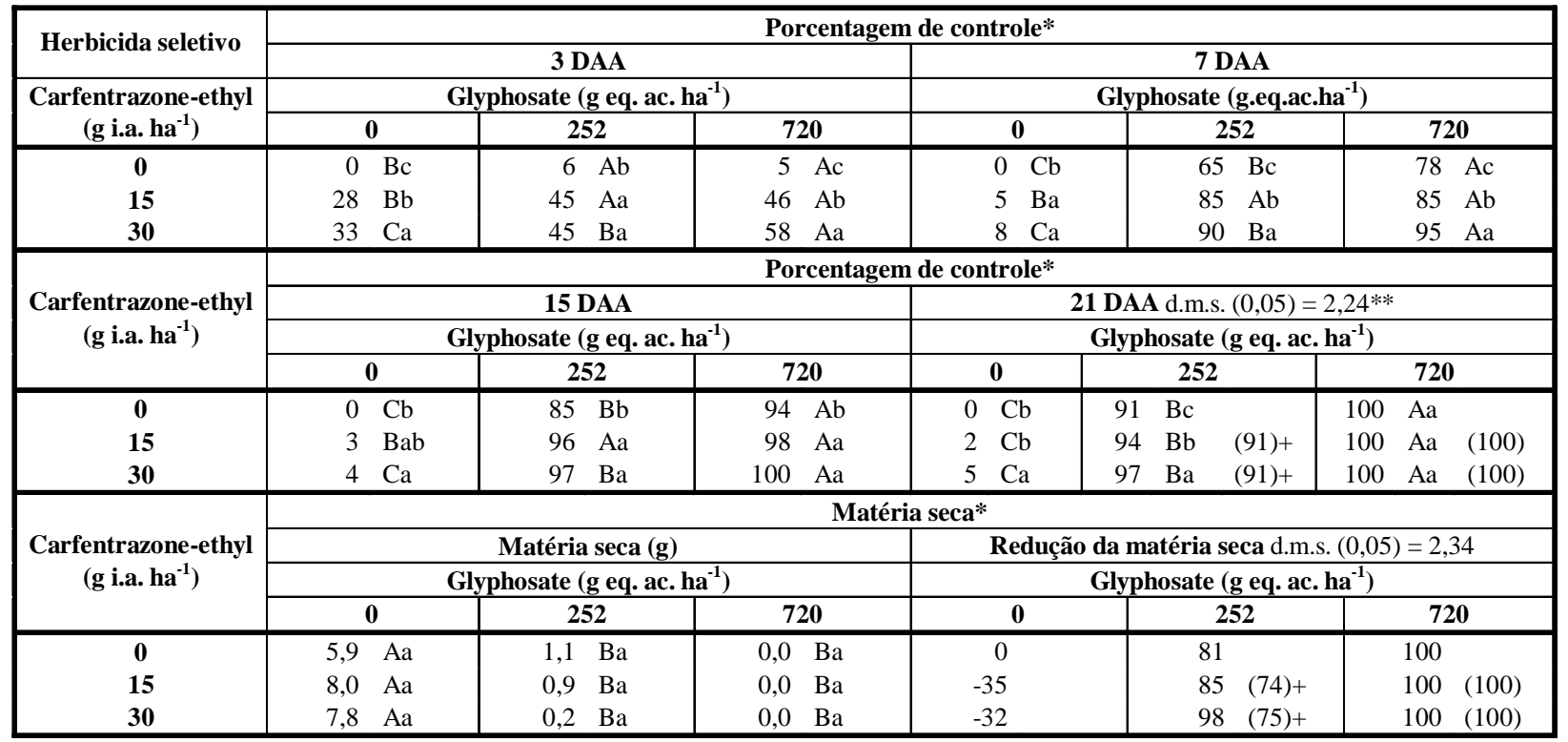

* Médias seguidas pelas mesmas letras, maiúsculas na linha e minúsculas na coluna, não diferem entre si pelo teste de Tukey a 5\% de significância.

** Valores entre parênteses são os valores esperados calculados pela equação de Colby (1967). O sinal negativo após o valor esperado indica efeito antagônico na interação, sinal positivo significa efeito de sinergismo e ausência de sinal indica interação aditiva. 
Tabela 7 - Controle visual, redução da matéria seca avaliada aos 21 DAA e esperado (Colby) de Commelina benghalensis, após aplicação de herbicidas em pós-emergência. Viçosa-MG, 2001

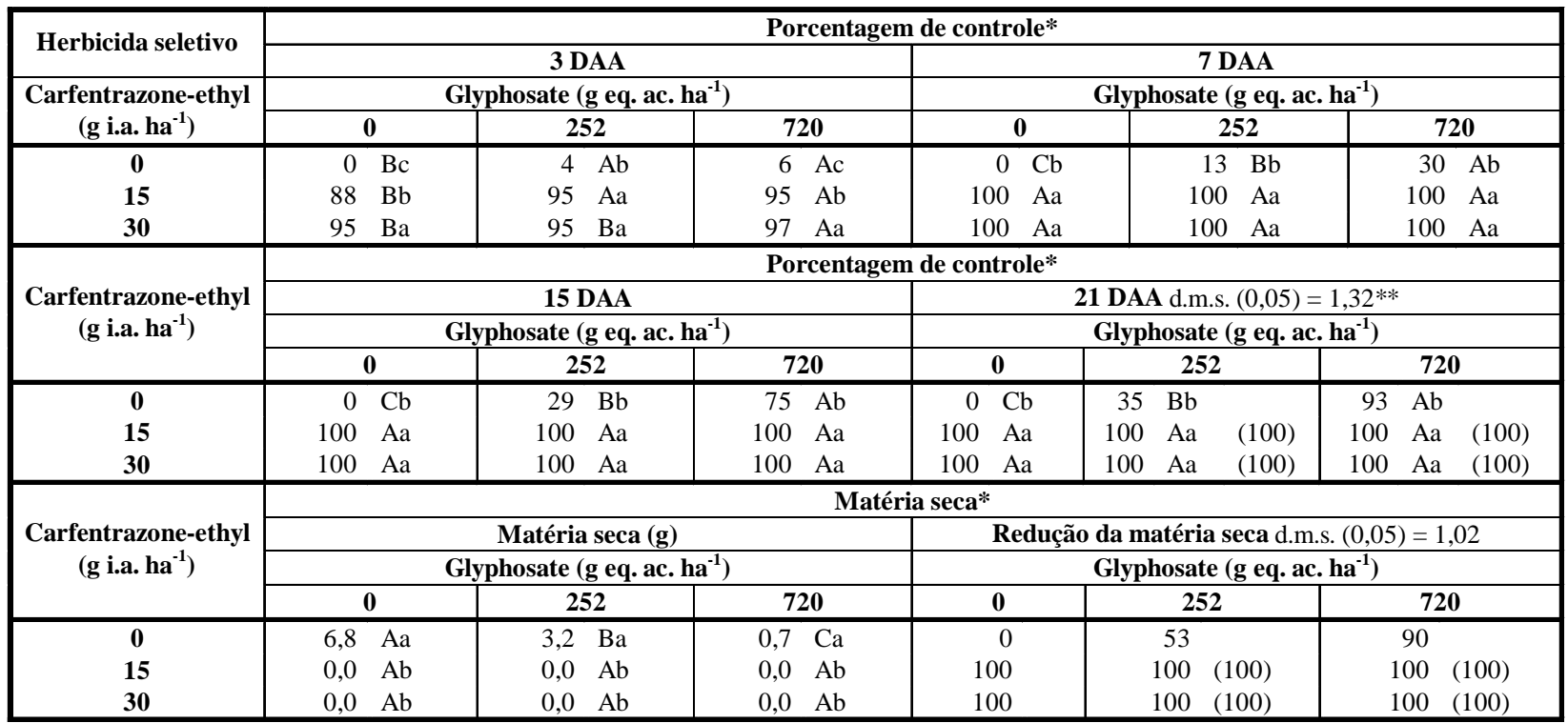

* Médias seguidas pelas mesmas letras, maiúsculas na linha e minúsculas na coluna, não diferem entre si pelo teste de Tukey a 5\% de significância.

** Valores entre parênteses são os valores esperados calculados pela equação de Colby (1967). O sinal negativo após o valor esperado indica efeito antagônico na interação, sinal positivo significa efeito de sinergismo e ausência de sinal indica interação aditiva.

Quanto ao efeito ocasionado nas gramíneas, a adição das duas doses de carfentrazone-ethyl antagonizou o efeito de glyphosate na menor dose (252 $\left.\mathrm{g} \mathrm{ha}^{-1}\right)$ no controle visual e redução de matéria seca de E. indica; no entanto, glyphosate na dose de $720 \mathrm{~g} \mathrm{ha}^{-1}$ proporcionou efeito aditivo misturado com as duas doses de carfentrazone-ethyl. O incremento na dose de glyphosate eliminou o efeito antagônico, semelhantemente ao observado por Jordan et al. (1997) com a mistura de glyphosate com acifluorfen. Para D. horizontalis, as misturas de carfentrazone-ethyl com glyphosate na menor dose (252 $\mathrm{g} \mathrm{ha}^{-1}$ ) apresentaram efeito sinergístico no controle visual e redução da matéria seca desta espécie, demonstrando, ainda, efeito aditivo na mistura com glyphosate na dose de $720 \mathrm{~g} \mathrm{ha}^{-1}$.

A mistura de carfentrazone-ethyl com glyphosate proporcionou efeito aditivo no controle de $C$. benghalensis, independentemente das combinações de doses avaliadas.

Os resultados deste experimento indicam que carfentrazone-ethyl apresenta comportamento diferenciado quanto à interação com glyphosate, dependendo da espécie de planta daninha e da dose dos herbicidas utilizados na mistura em tanque, sendo compativel na mistura em tanque com glyphosate, pois demonstrou efeito antagônico em poucas das combinações estudadas, prevalecendo seu efeito aditivo na mistura com glyphosate, no controle das espécies avaliadas.

Foram verificados, neste estudo, resultados semelhantes aos observados por Lee et al. (1997), que relataram controle excelente com a mistura de carfentrazone-ethyl $\left(25 \mathrm{~g} \mathrm{ha}^{-1}\right)+$ glyphosate ( $1.230 \mathrm{~g} \mathrm{ha}^{-1}$ ) em Artemisia princeps, Chenopodium album, Polyganum hydropiper, Erigeron canadensis, Commelina communis, Calystegia japonica, Amaranthus ascendents, Digitaria sanguinalis, Setaria viridis e Echinochloa crus-galli.

Carfentrazone-ethyl é uma molécula de $\mathrm{N}$-fenil-heterociclo, sendo semelhante à molécula dos herbicidas difeniléteres; possivelmente este herbicida tenha as mesmas características atribuídas ao lactofen (difeniléter), quando misturado com glyphosate em pequenas doses. Segundo Wells \& Appleby (1992), lactofen 
interage com glyphosate melhorando a atividade deste na planta, por facilitar a movimentação de glyphosate via simplasto na planta. Lactofen em doses pequenas melhorou a captura de glyphosate para o interior celular. No entanto, esta ação é pouco relatada na literatura, possivelmente devido às doses mais altas utilizadas em campo, sendo comum o antagonismo da ação de glyphosate, quando em mistura (Jordan et al., 1997; Starke \& Oliver, 1998).

Carfentrazone-ethyl, uma vez absorvido pelas folhas, vai inibir a formação da enzima protoporfirinogênio oxidase (PPO), que participa na sintese da clorofila; entretanto, na presença de luz ocorre a ruptura das membranas celulares, causando rápida e irreversível necrose dos tecidos foliares, as quais podem atrapalhar a absorção de outros herbicidas. Os efeitos antagônicos da mistura de carfentrazone-ethyl com glyphosate foram observados, principalmente, no controle de Desmodium tortuosum e Eleusine indica. Esse efeito antagônico da mistura de herbicidas que possuam ação de contato com o glyphosate foi previamente relatado (Selleck \& Baird, 1981; Hydrick \& Shaw, 1994; Lich et al., 1997; Starke \& Oliver, 1998). Segundo Jordan et al. (1997) e Starke \& Oliver (1998), a redução no controle das plantas daninhas pela mistura de glyphosate com um herbicida que possua ação de contato se deve à redução da quantidade de glyphosate absorvido e translocado na planta.

\section{LITERATURA CITADA}

CHRISTOFFOLETI, P. J.; VICTORIA FILHO, R.; SILVA, C. B. Resistência de plantas daninhas aos herbicidas. Planta Daninha, v. 12, n. 1, p. 13-20, 1994.

COLBY, S. R. Calculating synergistic and antagonistic responses of herbicide combinations. Weeds, v. 15, p. 20-22, 1967.

CORRÊA, L. E. A.; BORGES, A. Glyphosate + carfentrazone: controle de ervas problemas. In: CONGRESSO BRASILEIRO DA CIÊNCIA DAS PLANTAS DANINHAS, 22, 2000, Foz do Iguaçu. Resumos... Londrina: Sociedade Brasileira da Ciência das Plantas Daninhas, 2000. p. 463.

DELANNAY, X. et al. Yield evaluation of a glyphosatetolerant soybean line after treatment with glyphosate. Crop Sci., v. 35, p. 1461-1467, 1995.
DURIGAN, J. C.; GALLI, A. J. B.; LEITE, G. J. Avaliação da eficiência da mistura de glyphosate e 2,4-D para o controle de plantas daninhas em citrus. In: CONGRESSO BRASILEIRO DE HERBICIDAS E PLANTAS DANINHAS, 17, 1988, Piracicaba. Resumos... Piracicaba: Sociedade Brasileira da Ciência das Plantas Daninhas, 1988. p. 303-304.

FMC Corp. Boletim Técnico do Aurora 400 CE (carfentrazone ethyl). 16 p. 2001.

GALLI, A. J. B. Avaliação da eficiência de glyphosate em mistura com diversos produtos, no controle de Commelina virginica (trapoeraba) em citros. In: CONGRESSO BRASILEIRO DE HERBICIDAS E PLANTAS DANINHAS, 18, 1991, Brasília. Resumos... Brasília: Sociedade Brasileira da Ciência das Plantas Daninhas, 1991. p. 104-105.

GARCIA, I.; NASCIMENTO, E. Avaliação do herbicida carfentrazone-ethyl (Aurora $400 \mathrm{CE}$ ) em mistura com glyphosate e sulfosate no controle de plantas daninhas em semeadura direta. In: CONGRESSO BRASILEIRO DA CIÊNCIA DAS PLANTAS DANINHAS, 22, 2000, Foz do Iguaçu. Resumos... Londrina: Sociedade Brasileira da Ciência das Plantas Daninhas, 2000. p. 121.

GAZZIERO, D. L. P. et al. Herbicide alternatives for 2,4-D in no-till cropping systems. In: INTERNATIONAL WEED SCIENCE CONGRESS-IWSC, 2000, Foz do Iguaçu. Proceedings... Foz do Iguaçu: 2000. p.134.

HATZIOS, K. K.; PENNER, D. Interaction of herbicides with other agrochemicals in higher plants. Rev. Weed Sci., v. 1, p. 1-63, 1985.

HESS, F. D. Mechanism of action of inhibitors of amino acid biosynthesis. In: HERBICIDE action: an intensive course on the activity, seletivity, behavior, and fate of herbicides in plants and soil. West Lafayette: Purdue University, 1994. p. 344-365.

HYDRICK, D. E.; SHAW, D. R. Effects of tank-mix combinations of non-seletive foliar and selective soilapplied herbicides on three weed species. Weed Technol., v. 8, p. 129-133, 1994.

JORDAN, D. L. et al. Influence of application variables on efficacy of glyphosate. Weed Technol., v. 11, p. 354-362, 1997.

KAPUSTA, G. R.; KRAUSZ, R. F.; MATTHEWS, J. L. Soybean tolerance and summer annual weed control with glufosinate and glyphosate in resistent soybean. Proc. N. Cent. Weed Control Conf., v. 49, p. 120, 1994.

Planta Daninha, Viçosa-MG, v.20, n.1, p.93-102, 2002 
LEE, H. J. et al. Evaluation of carfentrazone-ethyl alone and in combination with glyphosate or glufosinate for weed control in orchards. Kor. J. Weed Sci., v. 17, n. 3, p. 256-261, 1997.

LICH, J. M.; RENNER, K. A.; PENNER, D. Interaction of glyphosate with postemergence soybean (Glycine max) herbicides. Weed Sci., v. 45, p. 12-21, 1997.

MALIK, J.; BARRY, G; KISHORE, G. The herbicide glyphosate. Biofactores, v. 2, p. 17-25, 1989.

OLIVEIRA Jr., R. S. et al. Carfentrazone: novo herbicida para o manejo de Ipomoea grandifolia e Commelina benghalensis em áreas de semeadura direta de soja e milho. In: CONGRESSO BRASILEIRO DA CIÊNCIA DAS PLANTAS DANINHAS, 22, 2000, Foz do Iguaçu. Resumos... Londrina: Sociedade Brasileira da Ciência das Plantas Daninhas, 2000. p. 440.

PEREIRA, W.; CRABTREE, G. Absorption, translocation, and toxicity of glyphosate and oxyfluorfen in yellow nutsedge (Cyperus esculentus). Weed Sci., v. 34, p. 923-929, 1986.

RODRIGUES, B. N.; ALMEIDA, F. S. Guia de herbicidas. 4.ed. Londrina: Edição dos Autores, 1998. $648 \mathrm{p}$.

RONCHI, C. P.; SILVA, A. A.; FERREIRA, L. R. Manejo de plantas daninhas em lavouras de café. Viçosa, MG: Suprema, 2001. 94 p.
SANTOS, I. C. et al. Eficiência de glyphosate no controle de Commelina benghalensis e Commelina diffusa. Planta Daninha, v. 19, n. 1, p. 135-143, 2001.

SELLECK, G. W.; BAIRD, D. D. Antagonism of glyphosate and residual herbicide combinations. Weed Sci., v. 29, p. 185-190, 1981.

SILVA, M. A.; COBUCCI, T. Herbicidas aplicados em préplantio no controle de plantas daninhas, na cultura do feijoeiro. In: CONGRESSO BRASILEIRO DA CIÊNCIA DAS PLANTAS DANINHAS, 22, 2000, Foz do Iguaçu. Resumos... Londrina: Sociedade Brasileira da Ciência das Plantas Daninhas, 2000. p. 235.

STARKE, R. J.; OLIVER, L. R. Interaction of glyphosate with chlorimuron, fomesafen, imazethapyr, and sulfentrazone. Weed Sci., v. 46, p. 652-660, 1998.

VARGAS, L. et al. Características da resistência de acordo com o mecanismo de ação herbicida. In: VARGAS, L. et al. (Eds.) Resistência de plantas daninhas a herbicidas. Viçosa: Universidade Federal de Viçosa, 1999. p. 16-34.

VIDRINE, P. R. et al. Post-emergence weed control in soybeans using glyphosate and chlorimuron. Proc. South. Weed Sci. Soc., v. 50, p. 175, 1997.

WELLS, B. H.; APPLEBY, A. P. Lactofen increases glyphosate-simulated shikimate production in little mallow (Malva parviflora). Weed Sci., v. 40, p. 171-173, 1992. 